\title{
Light-controllable viscoelastic properties of a photolabile carboxybetaine ester-based polymer with mucus and cellulose sulfate
}

\author{
Miroslav Mrlik ${ }^{1} \cdot$ P. Sobolciak ${ }^{2} \cdot$ I. Krupa $^{2} \cdot$ Peter Kasak $^{2}$ (D) \\ Received: 16 February 2018 / Accepted: 7 June 2018 \\ (C) Springer International Publishing AG, part of Springer Nature 2018
}

\begin{abstract}
In this study, the interaction of a photoswitchable carboxybetaine ester-based polymer with mucus and cellulose sulfate was elucidated, showing light-controllable viscoelastic properties. This polymer contains photolabile $o$-nitrobenzyl ester moieties, allowing transformation from its polycationic form to a charge-balanced nontoxic polyzwitterionic form upon photolysis by irradiation at $365 \mathrm{~nm}$. Rheological studies revealed that the polycationic form of the polymer interacts with mucus and cellulose sulfate to create a physically crosslinked hydrogel based primarily on polyionic complexation and partially on hydrogen bonding. In these cases, a dramatic change in the rheological synergism was confirmed for mucus-based and cellulose sulfate-based systems. Rheological synergism with the polycationic carboxybetaine ester sample reached nearly 4 and 3.8, while it decreased with the charge-balanced zwitterionic sample to 0.3 and 0.7 after irradiation of the mucus-based and cellulose sulfate-based systems, respectively. Disruption of the interaction during light-induced transformation was on-line monitored and showed a 3 and 3.3 times decrease in the elastic modulus for the mucus-based and cellulose sulfate-based systems, respectively. These properties suggest possible biomedical applications, such as spatially controlled drug release or laparoscopic utilization.
\end{abstract}

Keywords Mucus $\cdot$ Cellulose sulfate $\cdot$ Photolabile polymers $\cdot$ Carboxybetaine ester $\cdot$ Zwitterionic polymers

\section{Introduction}

Utilization of materials possessing the ability to control interactions with bioactive species has received attention in the biomedical, bioengineering and pharmaceutical fields [1]. A main challenge in the formation and application of this type of material is the lack of safety, cost effectiveness, efficiency, and controllable triggers. The character of the interactions of these smart materials can be tuned by applying external stimuli such as $\mathrm{pH}$, temperature, light, ionic strength, or electrical or magnetic field. Application of light as a noninvasive trigger has been increasingly utilized since it can be initiated in a timely

Miroslav Mrlik

mrlik@utb.cz

Peter Kasak

peter.kasak@qu.edu.qa

1 Centre of Polymer Systems, University Institute, Tomas Bata University in Zlin, Trida T. Bati 5678, 76001 Zlin, Czech Republic

2 Center for Advanced Materials, Qatar University, P. O. Box 2713, Doha, Qatar and spatially located manner and is compatible highly delicate bioactive species [2-4].

Materials containing carboxybetaine ester moieties have received increased interest due to their ability to undergo chemical changes by hydrolysis or photolysis based on the ester group [5-8]. Carboxybetaine ester-based materials can change their chemical properties by controlled hydrolysis or photolysis from the cationic state to the charge-balanced zwitterionic state, and this transformation dramatically modifies interactions with biological species [9-11]. pH- and light-induced transformations have been applied $[12,13]$. Previously, we have demonstrated that a carboxybetaine ester-based polymer consisting of the photolabile o-nitrobenzyl ester moiety was capable of lighttriggered transformation under $365 \mathrm{~nm}$ irradiation and showed potential as a vehicle for controlled complexation and release of DNA upon exposure to light [13]. Moreover, it showed irreversible light-switching from the polycationic form with antibacterial properties to the nontoxic zwitterionic form when tested in solution and at the surface when employing the Gram-positive bacteria Escherichia coli, proving the good compatibility of this polymer with living cells.

Materials with stimuli-adjustable interactions with mucus, especially polymers, are widely used for pharmaceutical 
applications of drug delivery in mucosal areas such as the intestine, stomach, vaginal tract, and ocular mucosa [14]. Polymers for these purposes exhibit the appropriate interaction with mucus by, e.g., ionic interaction, physical entanglement, van der Waals forces, hydrogen bonds or electrostatic forces. [15-22]. However, the nature, concentration and molecular weight of the utilized polymer considerably affect the adhesive properties and consequently affect drug absorption $[23,24]$. Furthermore, drug delivery systems can consist of not only polymers with proper interactions with mucus but also polymers that adhere to cellulose derivatives [25, 26].

Cellulose sulfate, as a semisynthetic polysaccharide sulfate, possesses unique biological properties [27], such as anticoagulation [28] and antiviral properties [29] and cell regulation function [30,31]. Moreover, cellulose sulfate has been used in bioengineering applications by polyelectrolyte complexation for the microencapsulation of enzymes [32] or Langerhans islets [33].

Additionally, there is limited time for suitable interactions between the polymer and both the mucus and cellulose (substrates). Here, various aspects, such as the thickness of the substrate, cyclic changes, viscosity and $\mathrm{pH}$, play an important role [34, 35].

For these purposes, various methods were employed to evaluate the interaction of the polymers with the substrate. Tensile tests, as a representative assessment for ex vivo studies, were used due to their simplicity [36, 37]. Furthermore, rheological studies based on the viscosity measurement of polymer solutions in the absence and in the presence of mucus have been published [38]. Here, rheological synergism, which is a calculated parameter, expresses the strong interaction of the polymer with the substrate. Based on these findings, other authors started to utilize oscillatory shear measurements to study these interactions [39-42]. In this case, polymers were evaluated as a bioadhesive when the rheological response of the polymersubstrate mixture was higher than the response of both individual components [43-46]. Moreover, the rheological properties can be influenced by the concentration of the substrates, i.e., after various extractions, different concentrations of the substrate origins can be obtained [47-49], or by the dissolving media used for the hydrogel preparation $[39,50]$. All these attributes need to be taken into account if such systems are to be applied in drug delivery or for biomedical purposes.

This study is aimed to examine the interactions of a carboxybetaine ester-based polymer bearing the photolabile 2nitrophenyl ester group, namely, poly $(N, N$-dimethyl$N$-[3-(methacroylamino) propyl]-N-[2-[(2nitrophenyl)methoxy]-2-oxo-ethyl]ammonium chloride) (CBE), with mucus and cellulose sulfate as model systems. Additionally, from investigation of the viscoelastic properties of both species, the adhesion of $\mathrm{CBE}$ and its photolyzed zwitterionic carboxybetaine form, $\operatorname{poly}(N$-carboxymethyl- $N, N$-dimethyl- $N$-[3-methacryloylamino propyl] ammonium betaine),
(CB), on mucus or cellulose sulfate can be investigated. The relative rheological synergism, as a crucial parameter for this evaluation, was evaluated. Moreover, the light-controllable interaction of the transformation of carboxybetaine ester-based samples (CBE) to carboxybetaine polymers (CB) with mucus and cellulose sulfate was confirmed. The approaches allow the utilization of these systems as potential materials for drug delivery and for release or adhesion in surgical applications.

\section{Experimental}

\subsection{Materials}

Mucus from porcine stomach (Mucin, Sigma Aldrich), $0.01 \mathrm{M}$ phosphate buffer saline (PBS) $\mathrm{pH}=7.4$, cellulose sulfate (CS), and sodium salt were obtained from Acros Organics (Geel, Belgium). $N$-[3-(dimethylamino)propyl] methacrylamide (99\%, Sigma Aldrich), 2,2'-azobis(2-methyl propionitrile) (AIBN, 98\%, Sigma Aldrich), tetrahydrofuran (THF), and dimethylsulfoxide (DMSO) were purchased from Sigma Aldrich (USA) and used as received. $o$-Nitrobenzyl 2chloroacetate was prepared according to a modified procedure described in the literature [51].

Ultrapure water was obtained from an Ultrapure Water System NW Series system (Heal Force Bio-Meditech Holdings, Ltd., China). CBE polymer with an Mw of 54,700 g.mol ${ }^{-1}$ and dispersity of 2.0 was prepared according to a slightly modified procedure published elsewhere [13].

\subsection{Sample preparation}

First, $0,0.167,0.33,1,3,4,5$, and 6 wt. $\%$ of $\mathrm{CBE}$ or $\mathrm{CB}$ was dissolved in the corresponding amount of PBS. Then, $7.2 \mathrm{wt} . \%$ of mucus (M) was added to the polymer solution. After $1 \mathrm{~h}$ of mixing with a magnetic stirrer, a homogeneous sample was obtained. The prepared samples were made in triplicate, stored and equilibrated under $5{ }^{\circ} \mathrm{C}$ in a climatic chamber (Discovery 250, Italy) in order to avoid dehydration. The prepared samples are summarized in Table 1. Sample codes starting with CBE are the samples containing carboxybetaine ester state polymer, while sample codes starting with $\mathrm{CB}$ are samples of photolyzed carboxybetaine form of the synthesized polymer (Scheme 2). In the case of samples based on CS, a similar procedure was used for the sample preparation. Instead of mucus, CS was utilized.

\subsection{Rheological analysis}

The rheological properties of the samples were determined under oscillatory shear using a Bohlin Gemini rotational rheometer (Malvern Instruments, UK). The samples were placed into a Couette cell with a rotating inner cylinder of $14 \mathrm{~mm}$ in 
Table 1 Composition of prepared mucus-based samples

wt.\% of individual components in feed

\begin{tabular}{|c|c|c|c|c|c|c|c|c|}
\hline Sample code & $\mathrm{M}$ & CBE 0.167-M & $\begin{array}{l}\text { CBE0.33- } \\
\text { M }\end{array}$ & $\begin{array}{l}\text { CBE1- } \\
\text { M }\end{array}$ & $\begin{array}{l}\text { CBE3- } \\
M\end{array}$ & $\begin{array}{l}\text { CBE4- } \\
\text { M }\end{array}$ & $\begin{array}{l}\text { CBE5- } \\
M\end{array}$ & $\begin{array}{l}\text { CBE6- } \\
\text { M }\end{array}$ \\
\hline $\mathrm{CBE}$ & 0 & 0.167 & 0.33 & 1 & 3 & 4 & 5 & 6 \\
\hline M & 7.2 & 7.2 & 7.2 & 7.2 & 7.2 & 7.2 & 7.2 & 7.2 \\
\hline Sample code & $\mathrm{M}$ & CB 0.167-M & CB 0.33-M & CB 1-M & CB $3 \mathrm{M}$ & CB 4-M & CB 5-M & CB $6-<$ \\
\hline $\mathrm{CB}$ & 0 & 0.167 & 0.33 & 1 & 3 & 4 & 5 & 6 \\
\hline M & 7.2 & 7.2 & 7.2 & 7.2 & 7.2 & 7.2 & 7.2 & 7.2 \\
\hline
\end{tabular}

diameter and a stationary outer cylinder separated by a $0.7 \mathrm{~mm}$ gap. The test samples $(4 \mathrm{~mL})$ were loaded into the cylinder and all measurements were performed at $15^{\circ} \mathrm{C}$ due to the increased dehydration and degradation of the material at elevated temperatures [39].

The linear viscoelastic region (LVR) was determined from the dependence of the viscoelastic moduli (storage, $G^{\prime}$, and loss, $\left.G^{\prime \prime}\right)$ on the strain value $(\gamma)$ at an angular frequency of $6.28 \mathrm{rad}$. $\mathrm{s}^{-1}$. A value of $\gamma=0.28$ was chosen as the strain value in subsequent frequency sweep measurements. All samples were measured three times to ensure the reliability of the data.

\subsubsection{Shear-thinning investigations}

The measurements were performed at a constant frequency of $1 \mathrm{~Hz}$. The strain deformations were changed from 11 to $500 \%$. Individual measurements at a certain deformation lasted $120 \mathrm{~s}$. Together, 5 cycles were performed in order to prove the timedependent behavior, and in one cycle, two different deformations were evaluated. All measurements were also performed at a constant temperature of $15^{\circ} \mathrm{C}$.

\subsection{On-line monitoring of photolysis}

For the on-line monitoring of the light-induced transformation of the carboxybetaine ester-based polymer in systems with mucus or cellulose sulfate, linear viscoelastic conditions were used, where $\gamma=0.28$ and $0.7 \mathrm{~Hz}$. Then, the time dependence of the viscoelastic moduli was recorded, and their change proved the transformation of the $\mathrm{CBE}$ polymer form to the CB zwitterionic carboxybetaine form. A $365 \mathrm{~nm}$ OmniCure 1000s UV lamp (Excelitas Technologies, France) with an intensity of $8 \mathrm{~mW} \mathrm{~cm}^{-2}$ was used for UV deposition in the UV cell of an Anton Paar MCR-502 rotational rheometer (Anton Paar, Austria). All measurements were performed at $15^{\circ} \mathrm{C}$ in order to avoid dehydration, and a guarded ring was used as well. Around the geometry, a solution of PBS was dropped to provide a saturated atmosphere of evaporated water. Moreover, a temperature sensor was placed in the cell quite close to the sample, and no change in the temperature was recorded even at $0.1^{\circ} \mathrm{C}$.

\subsection{Polymer/substrate interaction evaluation}

Both viscoelastic moduli, storage modulus $G^{\prime}$ and loss modulus $G^{\prime \prime}$, were used as appropriate quantities for the evaluation of bioadhesive properties. $G^{\prime}$ represents the energy stored from the application of deformation to the material that can be recovered and reflects the elastic-like component in the material. On the other hand, $G^{\prime \prime}$ represents the energy lost from the application of deformation and reflects the viscouslike component of the material [41].

All samples were measured in the angular frequency range of $0.628-20 \mathrm{rad} \cdot \mathrm{s}^{-1}$. These measurements indicated the behavior of the samples in a relatively long period as the mucus usually occurs. The evaluations of $G^{\prime}$ and $G^{\prime \prime}$ were usually determined according to Mortazavi et al. [52]. Our determination was performed at $1.35 \mathrm{rad} \cdot \mathrm{s}^{-1}$, which is similar to the value used by Mortazavi et al. [53].

Furthermore, the viscoelastic behavior can also be described by the loss tangent, $\tan \delta$ [39]. Tan $\delta$ provides information on the overall viscoelasticity of the sample, calculated as $G^{\prime \prime} / G^{\prime}$. In the case of $\tan \delta>1$, viscous-like behavior dominates, while in the case of $\tan \delta<1$, elastic-like behavior prevails.

Synergism in rheological properties is often calculated as the difference in the viscoelastic quantities $\left(G^{\prime}\right.$ and $\left.G^{\prime \prime}\right)$ of the polymer-mucus mixture and the sum of the properties of the two separate components (Eq. 1) [40, 54].

$$
\begin{aligned}
\Delta G^{\prime} & =G_{\text {mix }}^{\prime}-\left(G_{\text {polymer }}^{\prime}+G_{\text {muсиs }}^{\prime}\right) \\
\Delta G^{\prime \prime} & =G_{\text {mix }}^{\prime \prime}-\left(G_{\text {polymer }}^{\prime \prime}+G_{\text {muсus }}^{\prime \prime}\right)
\end{aligned}
$$

In this case, the relative rheological synergism (Eq. 2), expressed as the relative increase in viscoelasticity, was used for the evaluation.

$$
\begin{aligned}
& \Delta G^{\prime} /\left(G^{\prime \prime} \text { polymer }+G^{\prime \prime}{ }_{\text {muси }}\right) \\
& \Delta G^{\prime \prime} /\left(G^{\prime \prime}{ }_{\text {polymer }}+G^{\prime \prime}{ }_{\text {mисиs }}\right)
\end{aligned}
$$




\section{Results and discussion}

\subsection{Polymer synthesis and photolysis}

The polymer CBE was prepared by a one-pot, 2-step synthesis as depicted in Scheme 1 [13]. Free-radical polymerization of $\mathrm{N}$-[3-(dimethylamino)propyl] methacrylamide was performed, followed by quaternization of poly( $N$-[3-(dimethylamino)propyl]methacrylamide) with $o$-nitrobenzyl 2-chlorooacetate in DMSO. The resulting polymer $\mathrm{CBE}$ is polycationic but contains the photolabile 2-nitrophenylmethyl ester group, which can endow CB with zwitterionic behavior after photolysis, as depicted in Scheme 2. Subsequent photolysis of the synthesized polymer was performed by using UV irradiation at $365 \mathrm{~nm}$ with an intensity of $8 \mathrm{~mW} \mathrm{~cm}^{-2}$ in the UV cell of a rotational rheometer as described in the section 2 . The zwitterionic form of the monomer unit is charge balanced, and the carboxylate anion shields the cationic group. It should be pointed out that the resulting carboxylate in the zwitterionic form is assumed to have a relatively low $\mathrm{p} K_{\mathrm{a}}$ due to the electron withdrawing character of the permanent quaternary ammonium group and should be close to that of $\left(\mathrm{CH}_{3}\right)_{3} \mathrm{~N}^{+} \mathrm{CH}_{2} \mathrm{COO}^{-}\left(\mathrm{p} K_{\mathrm{a}}=2.03\right)$ [55]. The principle of this dramatic change in the interacting monomer unit charge was further applied for the investigation of the viscoelastic properties with mucus and cellulose sulfate as model compounds.

\subsection{Viscoelastic properties evaluation with mucus}

First, the interaction of mucus with $\mathrm{CBE}$ was examined as schematically depicted in Scheme 3.

First, after mixing the two solutions of mucus and CBE, a highly viscous solution was formed, as confirmed by visual observation. The resulting material slowly moved after agitation. No precipitation or sedimentation was observed over the whole range of the studied concentrations of polymers and mucus. In the case of the photolyzed form $\mathrm{CB}$, the resulting material is less viscous and shows more liquid-like behavior.
As an example, the most concentrated mucus and polymer samples CBE6-M and CB6-M are shown in Scheme 3, demonstrating the possible interactions in the system. Different concentrations were used for investigation of the interaction of mucus with $\mathrm{CBE}$ and $\mathrm{CB}$, and the concentrations of the samples and their codes are listed in Table 1.

Representative viscoelastic measurements of the samples showed highly viscous behavior, as seen in Fig. 1. The obtained results were comparable to those observed for common mucus-CBE polymer systems based on poly(acrylic) acid $[56,57]$.

From the frequency dependence of the viscoelastic properties $\left(G^{\prime}, G^{\prime \prime}\right.$ and $\left.\tan \delta\right)$, enhanced elastic behavior can be seen for the sample containing more than $1 \mathrm{wt} . \%$ of polymer in the polymer-mucus mixture. Figure 1a shows that the values of $G^{\prime}$ increase with increasing polymer content due to increased interaction of the polymer chains with the glycoproteins of the mucus and reach nearly $10 \mathrm{~Pa}$ at $10 \mathrm{rad} \mathrm{s}^{-1}$. The relatively weaker $G^{\prime}$ of the samples may be ascribed to the unfavorable conformation of the polymer chain due to the restricted ability of the polymer to interact by hydrogen bonds [54]. A similar trend was observed for $G^{\prime \prime}$ in Fig. 1b, which also increases with increasing polymer content. Furthermore, from the dependence of $\tan \delta$ on $\omega$ shown in Fig. 1c, increased elasticity of the samples can be seen with increasing polymer content as the $\tan \delta$ reaches values of approximately 1.5 at $10 \mathrm{rad} \mathrm{s}^{-1}$.

On the other hand, when the photolyzed form of the polymer $\mathbf{C B}$ was used in the sample, the viscoelastic behavior is different (Fig. 2). The $G^{\prime}$ (Fig. 2a) only slightly increases as the amount of photolyzed polymer (CB) increases and reaches $5 \mathrm{~Pa}$ at $10 \mathrm{rad} \mathrm{s}^{-1}$, with the exception of CB 1-M, whose values are lower than those of neat mucus because of the change in the functional groups of this polymer, which are not able to sufficiently interact with the glycoproteins from mucus. The same behavior is observed from Fig. $2 \mathrm{~b}$ and c: $G^{\prime \prime}$ as well as $\tan \delta$ is not significantly influenced by the polymer content and in fact reaches values of approximately 1.8 at $10 \mathrm{rad} \mathrm{s}^{-1}$. This can be attributed to the strong hydration of carboxybetaine and the balanced charge in the same monomer unit shielding access to the charged moieties.
Scheme 1 Synthesis of the polymer CBE with a photolabile ester group
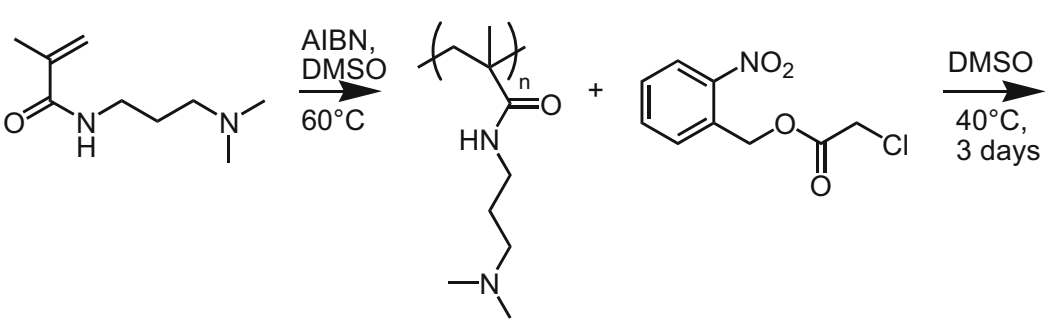

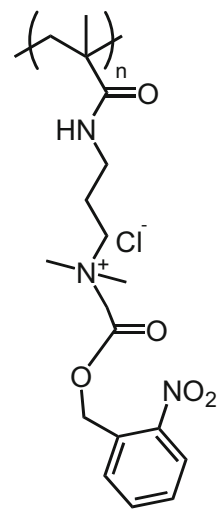


Scheme 2 Schematic presentation (sides) and chemical transformation (middle) of CBE to $\mathrm{CB}$ after photolysis under UV irradiation at $365 \mathrm{~nm}$

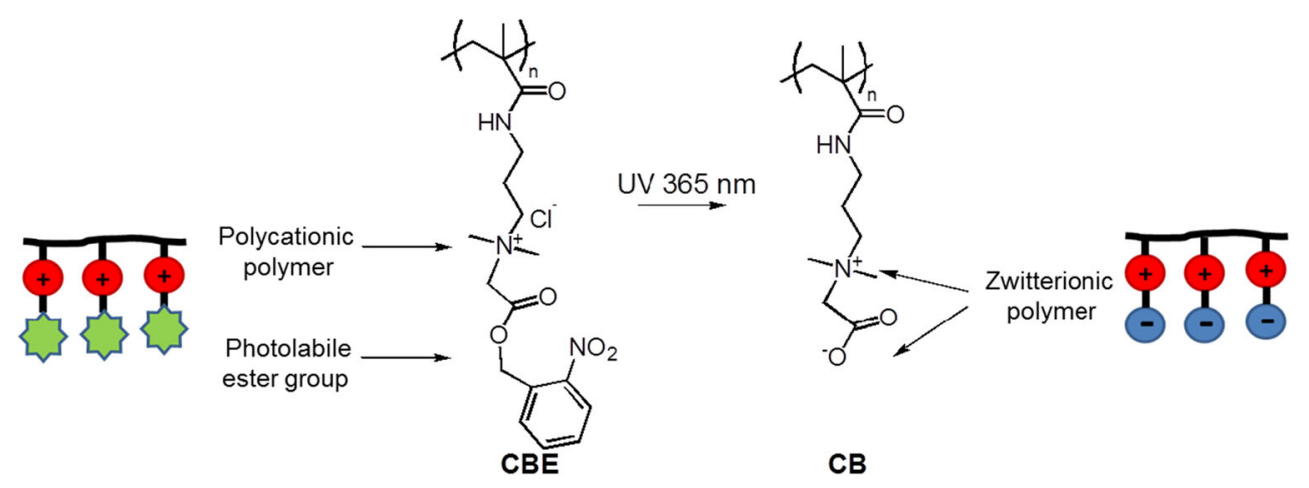

Finally, it is necessary to conclude that the samples containing the synthesized polymer CBE exhibit considerably higher interactions than the samples containing the photolyzed form $\mathbf{C B}$ due to the restricted ability of the polymer to interact with the glycoproteins of the mucus after photolysis.

\subsection{Viscoelastic properties evaluation with cellulose sulfate (CS)}

Similar concentrations of $\mathrm{CBE}$ and $\mathrm{CB}$ were used with the same concentration of CS as is schematically showed in Schem4 for investigating the interaction with CS, as summarized by the composition in the feed and sample codes in Table 2. Visual observation reveals the formation of a very viscous solution with $\mathrm{CS}$, which became less viscous after interaction with photolyzed CB. Scheme 4 middle shows digital images of samples containing CS with 6 wt.\% CBE (middle) and $\mathrm{CB}$ (right). Both samples are homogenous, and no visible precipitation or sedimentation is observed. Similarly, as with the mucus-based samples, the sample with $\mathrm{CB}$ is much less viscous due to shielding of the positive charge in $\mathrm{CB}$ by the negative charge of the zwitterionic form of the polymer. amount of the polymer in the sample is above $3 \mathrm{wt} . \%$
the liquid-like behavior of this system is still present.
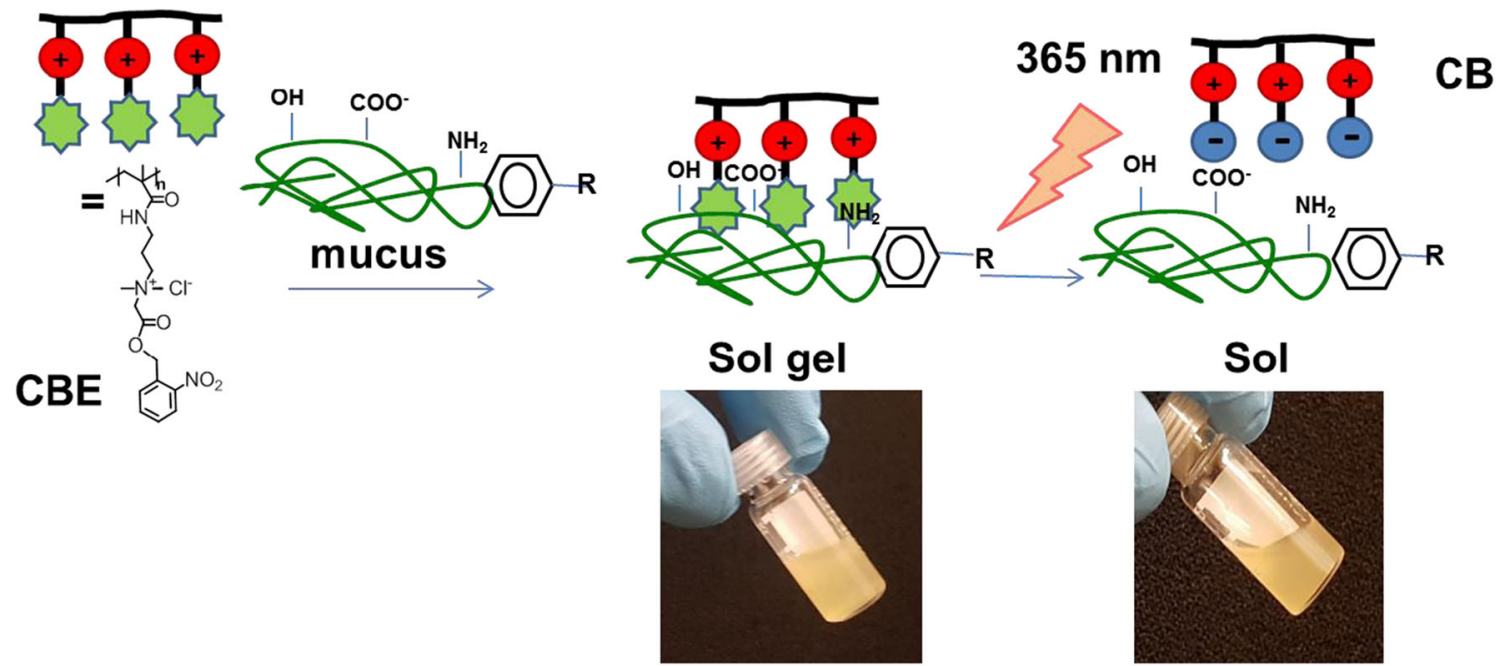

Scheme 3 Schematic presentation of interactions CBE and CBwith mucus and the images of sample CBE6-M (middle) and CB6-M (right) 


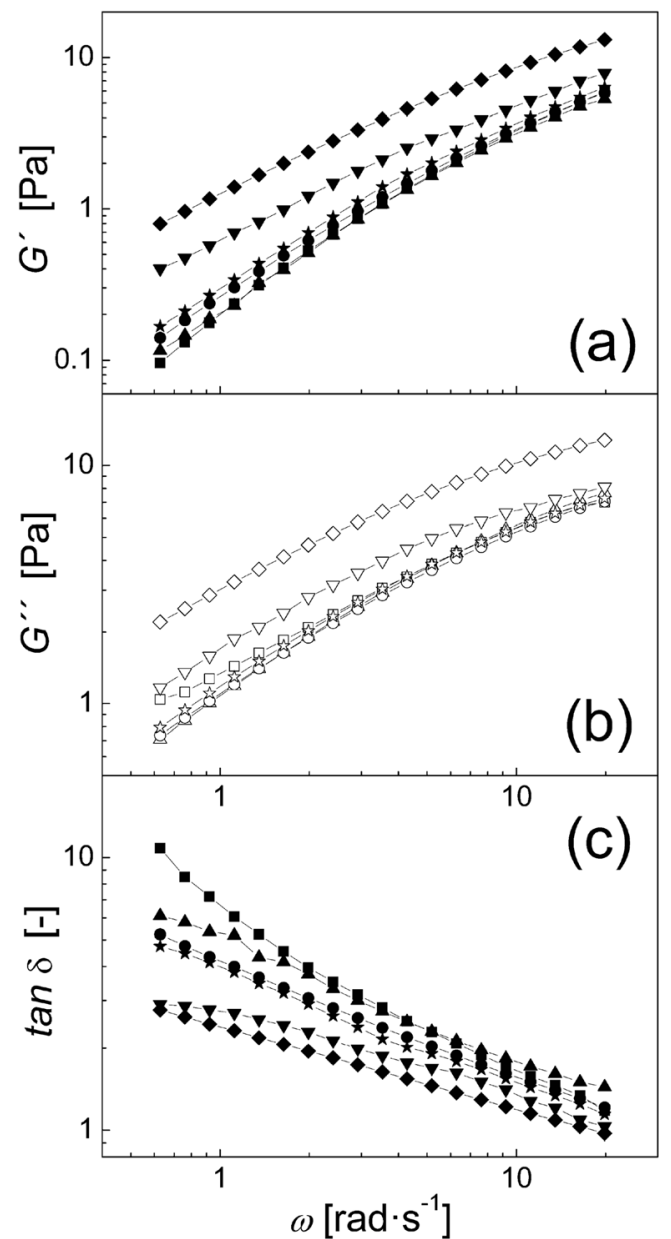

Fig. 1 Dependence of the elastic modulus $G^{\prime}$ (a), viscous modulus $G^{\prime \prime}(\mathbf{b})$ and $\tan \delta(\mathbf{c})$ on the angular frequency, $\omega$, for samples containing various concentrations of CBE: M $(\bullet, \square), \operatorname{CBE} 1-\mathrm{M}(\boldsymbol{\Delta}, \Delta), \mathrm{CBE} 3-\mathrm{M}(\bullet, \circ)$, CBE4-M ( $\star$, $\bullet)$, CBE5-M $(\boldsymbol{\nabla}, \nabla)$ and CBE6-M $(\bullet, \diamond)$

In the case of samples based on the interaction of CBE with $\mathrm{CS}$, the viscoelasticity obtained was similar to those observed for the mucus samples. However, in this case, the amount of polymer (sample CBE 0.33-CS) sufficient to exhibit strong interactions is lower than that of the sample without polymer (Fig. 4). Figure 4a clearly shows that the elastic modulus increases with increasing polymer content. In this case, the highest contribution to the elastic modulus is up to $4 \%$ of the polymer $\mathrm{CBE}$ in the sample. Above this polymer content, the elastic modulus increases only moderately but reaches values of over $400 \mathrm{~Pa}$ at $10 \mathrm{rad} \mathrm{s}^{-1}$. The viscous moduli of all samples (Fig. 4b) follow the behavior of the elastic one (increase with the polymer content). which means that no physical interactions similar to those observed in the case of mucus-based systems appear; instead, only dynamic ion-ion interactions and interactions through hydrogen bonding between the polymer chains and CS are responsible for the increases in both moduli [26], and the samples generally exhibit liquid-like rather than solid-like behavior, especially at low angular frequencies. As expected, the $\tan \delta$ (overall elasticity)

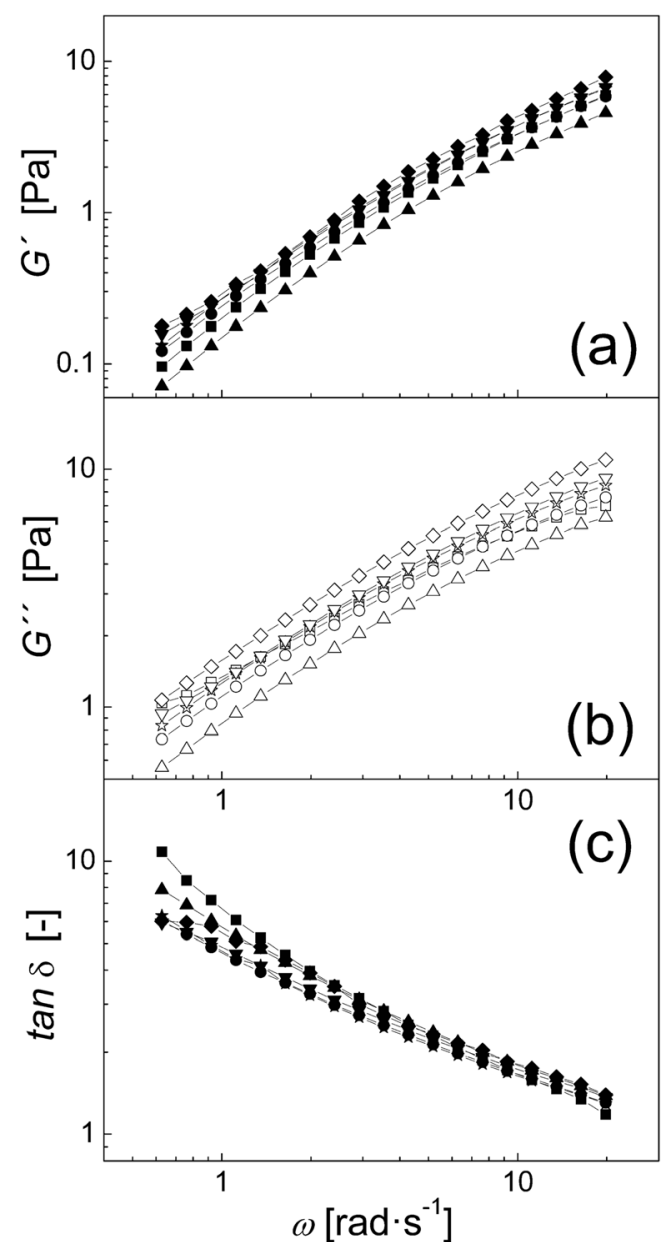

Fig. 2 Dependence of the elastic modulus $G^{\prime}(\mathbf{a})$, viscous modulus $G^{\prime \prime}(\mathbf{b})$ and $\tan \delta(\mathbf{c})$ on the angular frequency, $\omega$, for samples containing various concentrations of CB: M $(\bullet, \square)$, CB1-M $(\boldsymbol{\Delta}, \Delta)$, CB3-M $(\bullet, \circ)$, CB4-M $(\star, \star \star), \mathrm{CB} 5-\mathrm{M}(\boldsymbol{\nabla}, \nabla)$ and $\mathrm{CB} 6-\mathrm{M}(\bullet, \diamond)$. The line is a guide for eyes

decreases with polymer content (Fig. 4c). However, with increasing angular frequency, the $\tan \delta$ drops under 1 , indicating that solid-like behavior prevails and reaches values of 0.4 at $10 \mathrm{rad} \mathrm{s}^{-1}$. The interactions between polymer chains and CS are more pronounced for samples with a polymer content of more than $3 \%$. This result is further studied by evaluating the relative rheological synergism.

From the viscoelastic investigation of the samples based on CS and CB (Fig. 5), it can be seen that the elastic modulus increases with polymer content and that when above $3 \%$ polymer is present in the sample, negligible interactions are present, and the elastic modulus does not significantly increase at all.

For the proper investigation of the adhesive properties of the synthesized polymer and its photolyzed form, the relative rheological synergisms for both the elastic and viscous moduli are plotted against the polymer content (Fig. 6). As can be seen, the adhesive properties of the CBE-based samples were confirmed by the values reaching nearly 3.6 in the case of $G^{\prime}$ and by the weak adhesion of the CB-based samples after photolysis with values of approximately 0.7 . 


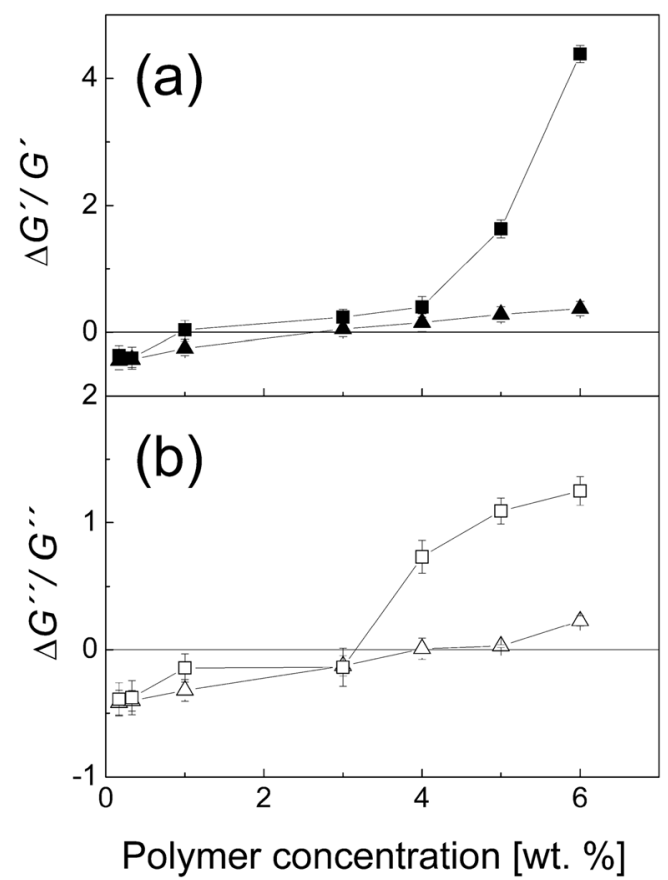

Fig. 3 Dependence of the relative rheological synergism of mucus-based systems for $\Delta \mathrm{G}^{\prime} / \mathrm{G}^{\prime}$ (a) and $\Delta \mathrm{G}^{\prime \prime} / \mathrm{G}^{\prime \prime}$ (b) on the polymer concentration of $\mathrm{CBE}(\boldsymbol{\square}, \square)$ and $\mathrm{CB}(\boldsymbol{\Delta}, \Delta)$. The lines are guides for the eyes

Here, it can be again stated that, after irradiation, the adhesive properties of the samples are considerably suppressed and that no interactions or, in the case of some samples, even weak interactions between polymer chains and the CS functional groups are present. Moreover, these kinds of samples thus can be utilized in applications with a controllable viscoelastic response.

\subsubsection{Shear-thinning investigations}

In the various applications targeted, the injection of polymeric systems is a very useful procedure to transport the system to the desired location. As seen in Fig. 7, the polymer-mucusbased systems exhibit rather negligible shear thinning, as $\mathrm{G}^{\prime}$ decreases only from 2.2 $\mathrm{Pa}$ to $1.3 \mathrm{~Pa}$ and $\mathrm{G}^{\prime \prime}$ decreases from 4.2 $\mathrm{Pa}$ to $3.5 \mathrm{~Pa}$ from 11 and $500 \%$ of strain deformation, respectively. On the other hand, the polymer-CS-based systems exhibit rather high shear-thinning behavior, as the $\mathrm{G}^{\prime}$ decreases from $70 \mathrm{~Pa}$ to $12 \mathrm{~Pa}$, and $\mathrm{G}^{\prime \prime}$ decreases from $103 \mathrm{~Pa}$ to $61 \mathrm{~Pa}$ for the same deformation regime. Such behavior is most likely caused by the quite weak ion-ion interaction in comparison to the polymer-mucus-based systems, where strong hydrogen bonding is most likely responsible for such behavior.

\subsubsection{On-line monitoring of photolysis}

To confirm that the already conducted rheological investigation can be applied in real-life applications, the on-line monitoring of the photolabile group cleavage was carried out (Fig. 8). For both the mucus (Fig. 8b) and CS systems (Fig. 8a), it can be seen that elastic modulus, representing the elastic behavior of the system, decreases with increasing time (from $28 \mathrm{~Pa}$ to $12.5 \mathrm{~Pa}$ and from 0.9 to 0.28 for the CS- and mucus-based systems, respectively) and that at $35 \mathrm{~min}$ of UV exposure, the plateau is nearly reached, at which point only a slight decrease of elastic modulus occurs. The time of irradiation for reaching the plateau of $35 \mathrm{~min}$ is shorter than the previously observed time needed for the release of DNA molecules from complexes on the $\mathrm{CBE}$ surface by plasmon resonance measurements (180 min) [13]. This can be attributed to the higher intensity of light used in the current case $\left(8 \mathrm{~mW} \mathrm{~cm}^{-2}\right)$ and the overall UV dose for $40 \mathrm{~min}$ of exposure being $19.2 \mathrm{~J} \mathrm{~cm}^{-2}$. Moreover, the effect of disrupted interactions on the viscoelastic properties is much faster than the effect of the removal of mass from the surface of the complexed material in a flow system.

Notably, experiments to prepare the CBE polymer on a $\mathrm{Au}$ surface for bioadhesion evaluation were attempted. Attempts to directly prepare the CBE polymer from its monomeric counterpart by means of either SI ATRP or SI free-radical polymerization with $N$-([3-(methacryloylamino)propyl]- $N, N$-dimethyl$N$-(2-nitrophenylmethylcarboxymethyl) ammonium chloride were also carried out, but polymeric brushes or thin layers were not obtained due to the polymerization inhibition effect of the nitro group in the ortho position of the monomer molecule [59]. Furthermore, experiments with post-polymerization

Table 2 Composition of prepared CS-based samples

\begin{tabular}{|c|c|c|c|c|c|c|c|c|}
\hline \multirow[b]{2}{*}{ Sample code } & \multicolumn{8}{|c|}{ wt. $\%$ of individual components } \\
\hline & $\mathrm{CS}$ & $\begin{array}{l}\text { CBE0.167- } \\
\text { CS }\end{array}$ & CBE0.33- CS & $\begin{array}{l}\text { CBE1- } \\
\text { CS }\end{array}$ & $\begin{array}{l}\text { CBE3- } \\
\text { CS }\end{array}$ & $\begin{array}{l}\text { CBE4- } \\
\text { CS }\end{array}$ & $\begin{array}{l}\text { CBE5- } \\
\text { CS }\end{array}$ & $\begin{array}{l}\text { CBE6- } \\
\text { CS }\end{array}$ \\
\hline Polymer & 0 & 0.167 & 0.33 & 1 & 3 & 4 & 5 & 6 \\
\hline M & 7.2 & 7.2 & 7.2 & 7.2 & 7.2 & 7.2 & 7.2 & 7.2 \\
\hline Sample code & $\mathrm{CS}$ & CB0.167-CS- & CB0.33-CS- & CB1-CS & CB 3CS & $\mathrm{CB}-4 \mathrm{CS}$ & CB5CS & CB6CS \\
\hline $\mathrm{CB}$ & 0 & 0.167 & 0.33 & 1 & 3 & 4 & 5 & 6 \\
\hline $\mathrm{CS}$ & 7.2 & 7.2 & 7.2 & 7.2 & 7.2 & 7.2 & 7.2 & 7.2 \\
\hline
\end{tabular}



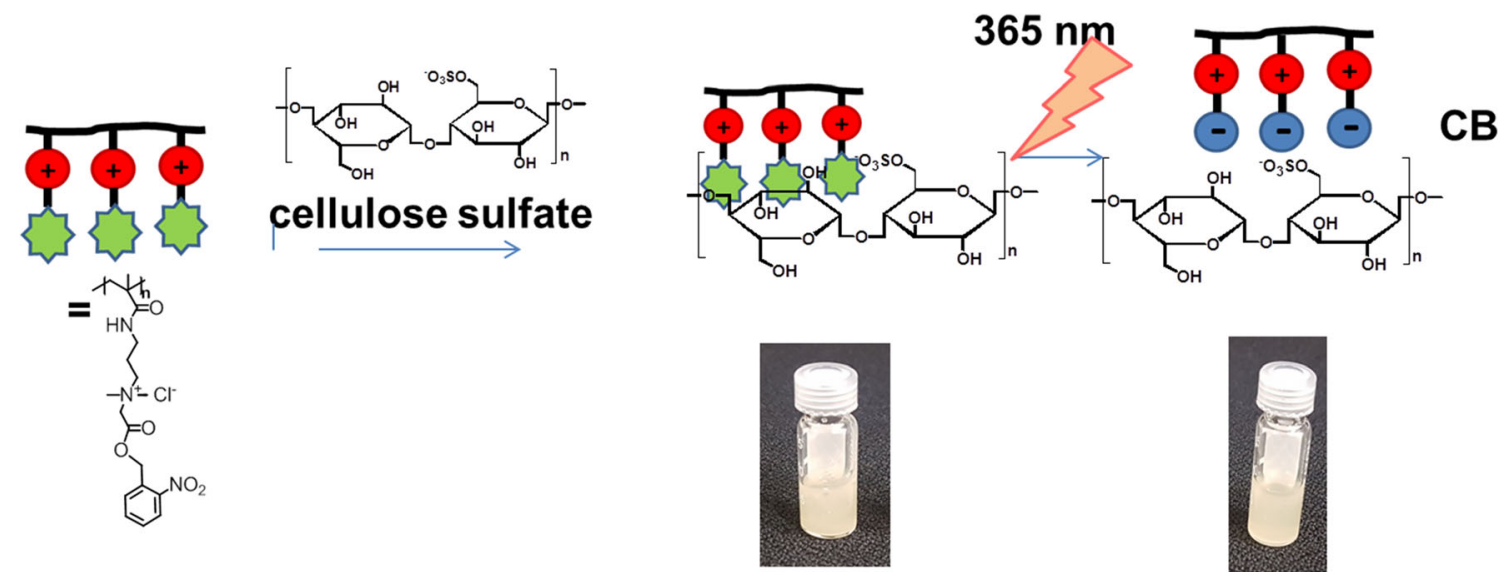

Scheme 4 Schematic presentation of interactions CBE and CB with cellulose sulfate and the images of sample CBE6-CS (middle) and CB6-CS (right)

modification of poly( $N$-[3-(dimethylamino)propyl] methacrylamide) were performed; however, quaternization was achieved in $16 \%$ yield as per XPS analysis. Nevertheless, the proposed applications do not require CBE polymer surface attachment, and the facile implementation of this concept in the already mentioned medical applications has been proven.

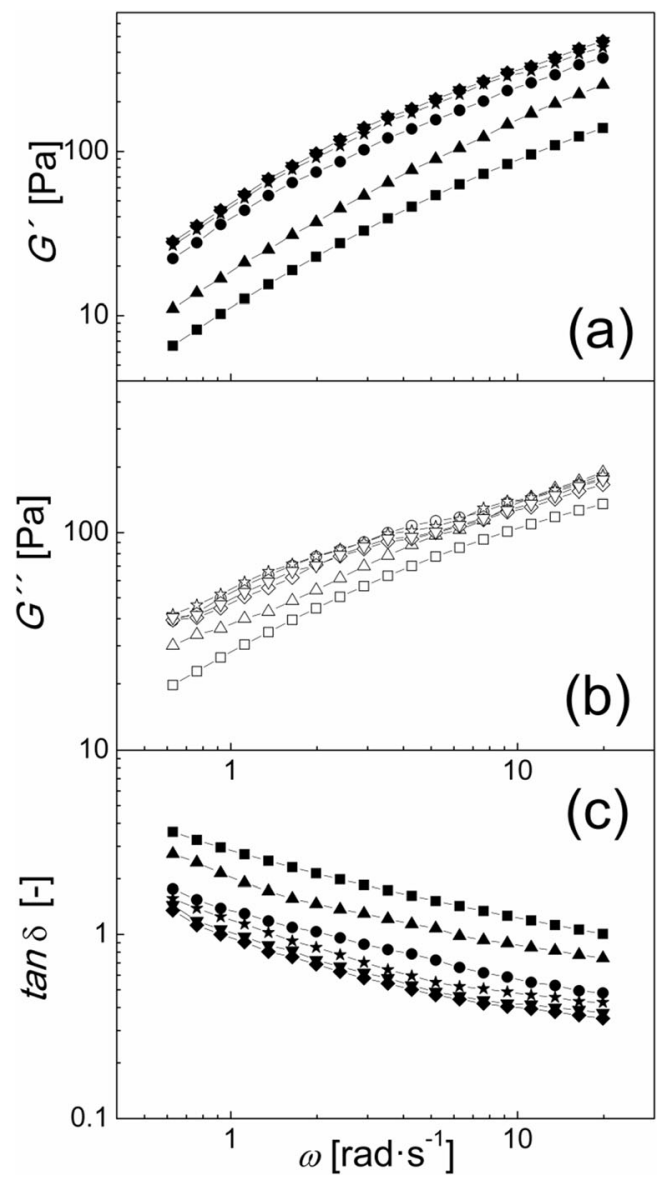

Fig. 4 Dependence of the elastic modulus $G^{\prime}\left(\right.$ a), viscous modulus $G^{\prime \prime}$ (b) and $\tan \delta$ (c) on the angular frequency, $\omega$, for samples containing various concentrations of CBE: CS $(\boldsymbol{\bullet}, \square), \mathrm{CBE}-\mathrm{CS} 1(\boldsymbol{\Delta}, \Delta), \mathrm{CBE} 3-\mathrm{CS}$ $(\bullet, \circ), \mathrm{CBE} 4-\mathrm{CS}(\star, \star)), \mathrm{CBE} 5-\mathrm{CS}(\boldsymbol{\nabla}, \nabla)$ and CBE6-CS $(\bullet, \diamond)$

\section{Conclusions}

This article extended our previous finding on photoswitchable polycationic CBE to charge-balanced polycarboxybetaine $\mathrm{CB}$, which showed dramatic changes in the interactions with DNA and bacteria after the

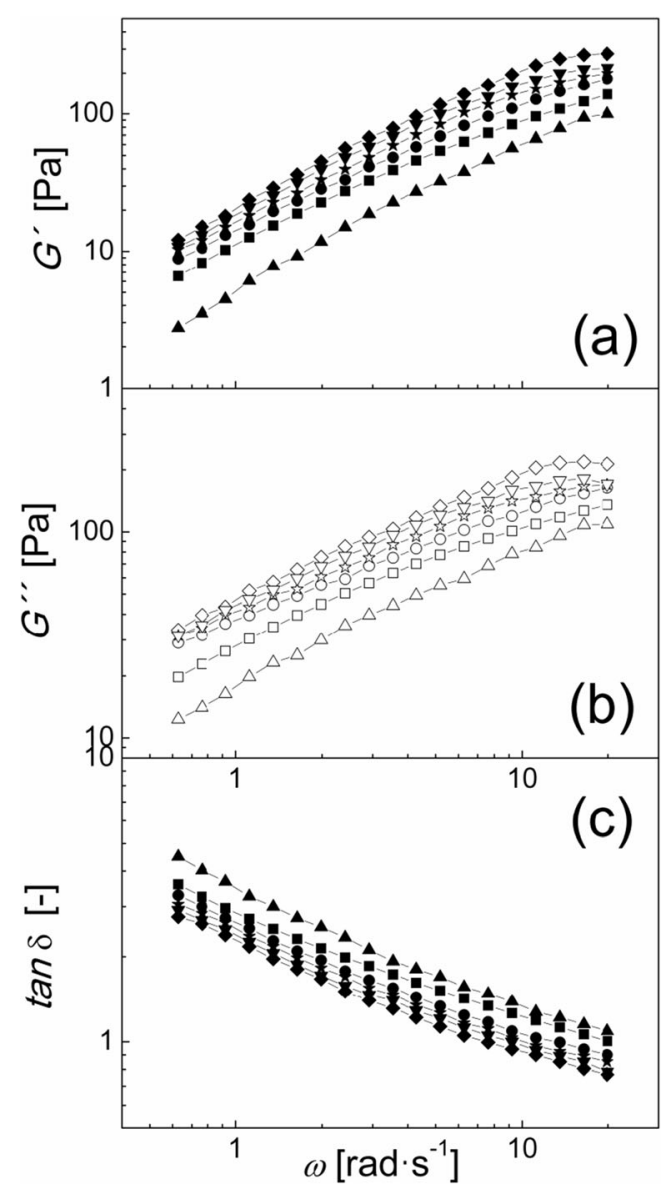

Fig. 5 Dependence of the elastic modulus $G^{\prime}\left(\right.$ a), viscous modulus $G^{\prime \prime}$ (b) and $\tan \delta$ (c) on the angular frequency, $\omega$, for samples containing various concentrations of CB: CS $(\bullet, \square)$, CB1-CS $(\boldsymbol{\Delta}, \Delta)$, CB3-CS $(\bullet$, $\circ), \mathrm{CB} 4-\mathrm{CS}(\star, \star)), \mathrm{CB} 5-\mathrm{CS}(\boldsymbol{\nabla}, \nabla)$ and CB6-CS $(\bullet, \diamond)$ 


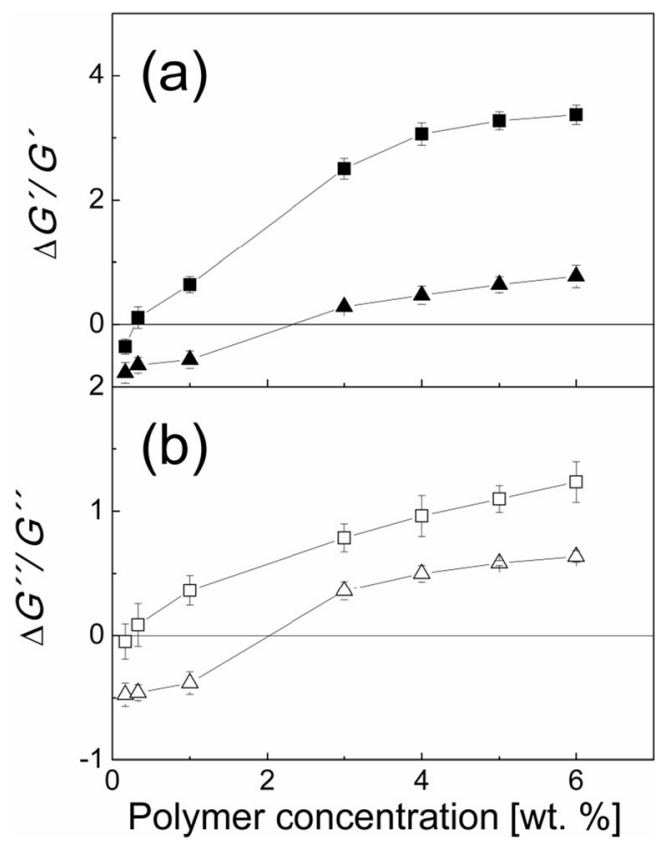

Fig. 6 Dependence of the relative rheological synergisms of CS systems for $\Delta \mathrm{G}^{\prime} / \mathrm{G}^{\prime}$ (a) and $\Delta \mathrm{G}^{\prime \prime} / \mathrm{G}^{\prime \prime}(\mathbf{b})$ on the polymer concentration of CBE ( ㅁ) and $\mathrm{CB}(\boldsymbol{\Delta}, \Delta)$

transformation [13]. This investigation revealed the controlled viscoelastic behavior of the polymer, exhibiting photoswitchable properties with bioactive molecules, which can be utilized in various medical applications. Through investigation of the viscoelastic properties, the interactions of polymer-based samples with both mucus and cellulose sulfate substrates were evaluated. The rheological synergism was in the same range as that with polyallylamine acid [56] or other systems based on chitosan [57]. Moreover, the viscoelastic properties can be modulated, and the interactions with both substrates were suppressed by UV light due to presence of the photolyzable ester group in $\mathrm{CBE}$, which makes this

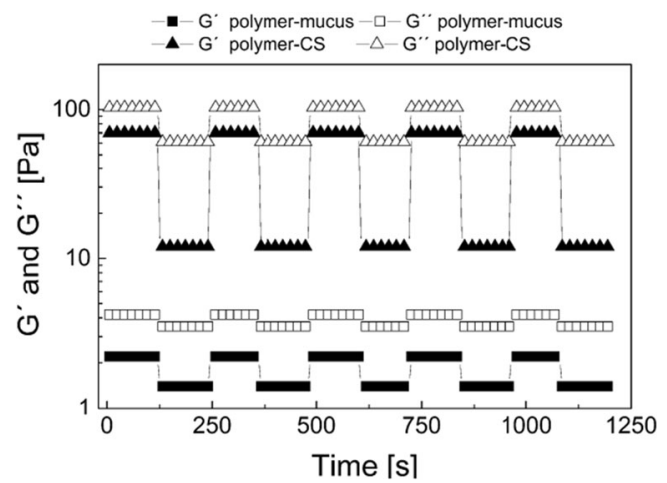

Fig. 7 Dependence of the storage and loss moduli on time at two regimes. The lower values always correspond to $500 \%$, and higher values correspond to $11 \%$ of strain deformation. All measurements were performed at $1 \mathrm{~Hz}$ and $15^{\circ} \mathrm{C}$

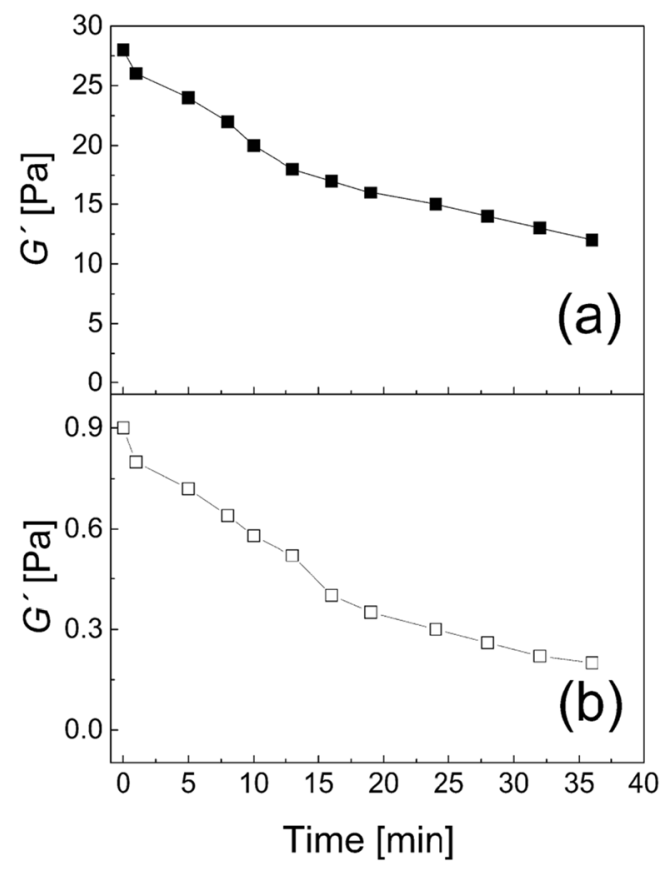

Fig. 8 On-line monitoring of G ' value during UV irradiation at $365 \mathrm{~nm}$ as a result of the transformation of $\mathrm{CBE}$ to $\mathrm{CB}$ in the (a) cellulose sulfateand (b) mucus-based systems

approach more attractive. Both investigated systems exhibited shear-thinning behavior, and CS showed better shear-thinning performance than the mucus CBE-systems. Prolonged photolysis led to a continual decrease in favorable interactions within sample networks due to the formation of the electronically balanced, nontoxic and hemocompatible zwitterionic form, which increases the rate of the disassembly and release of DNA from DNA-CBE complexes on the surface [13]. It should be pointed out that this study shows a proof of concept for the light-controlled adjustment of viscoelastic properties with biological active species; however, long-term UV light exposure may cause damage to biosystems. Thus, further studies are in progress to improve the photolysis by employing ester groups suitable for two-photon absorption.

Nevertheless, the light-modulated interactions of betaine-based polymers with various substrates open an avenue for spatial and temporal control of the modulation of viscoelastic properties, release or adhesion. This approach can be potentially used in bioactive species delivery or in surgical applications.

Acknowledgements The authors thank Dr. I. Lacik (Polymer Institute, the Slovak Academy of Science, Slovakia) for generously providing the laboratory facilities in the initial stage of the investigation. This publication was supported by Qatar University Grant QUUG-CAM-2017-1. This work was made possible by NPRP grant No. 7 - $1724-3$ - 438 from the Qatar National Research Fund (a member of The Qatar Foundation). The 
statements made herein are solely the responsibility of the authors. This work was also supported by the Ministry of Education, Youth and Sports of the Czech Republic - program NPU I (LO1504).

\section{References}

1. S. Khanlari, M.A. Dubé, Bioadhesives: a review. Macromol. React. Eng. 7, 573-587 (2013)

2. J. Cui, V. San Miguel, A. del Campo, Light-triggered multifunctionality at surfaces mediated by photolabile protecting groups. Macromol. Rapid Commun. 34, 310-329 (2013)

3. Y. Chen, Z. Wang, Y. He, Y.J. Yoon, J. Jung, G. Zhang, Z. Lin, Light-enabled reversible self-assembly and tunable optical properties of stable hairy nanoparticles. PNAS 115, E1391-E1400 (2018)

4. P. Klán, T. Šolomek, C.G. Bochet, A. Blanc, R. Givens, M. Rubina, V. Popik, A. Kostikov, J. Wirz, Photoremovable protecting groups in chemistry and biology: reaction mechanisms and efficacy. Chem. Rev. 113, 119-191 (2013)

5. Z. Zhang, G. Cheng, L.R. Carr, H. Vaisocherova, S. Chen, S. Jiang, The hydrolysis of cationic polycarboxybetaine esters to zwitterionic polycarboxybetaines with controlled properties. Biomaterials 29, 4719-4725 (2008)

6. B. Cao, Q. Tang, L.L. Li, J. Humble, H.Y. Wu, L.Y. Liu, G. Cheng, Switchable antimicrobial and antifouling hydrogels with enhanced mechanical properties. Adv. Healthc. Mater. 2, 1096-1102 (2013)

7. Z.Q. Cao, L. Mi, J. Mendiola, J.R. Ella-Menye, L. Zhang, H. Xue, S.Y. Jiang, Reversibly switching the function of a surface between attacking and defending against bacteria. Angew. Chem. Int. Ed. 51, 2602-2605 (2012)

8. B. Cao, L.L. Li, Q. Tang, G. Cheng, The impact of structure on elasticity, switchability, stability and functionality of an all-in-one carboxybetaine elastomer. Biomaterials 34, 7592-7600 (2013)

9. M. Ilcikova, J. Tkac, P. Kasak, Switchable materials containing polyzwitterion moieties. Polymers 7, 2344-2370 (2015)

10. J. Filip, A. Popelka, T. Bertok, A. Holazova, J. Osicka, J. Kollar, M. Ilčiková, J. Tkac, P. Kasak, pH-switchable interaction of a carboxybetaine ester-based SAM with DNA and gold nanoparticles. Langmuir 33, 6657-6666 (2017)

11. L. Mi, S.Y. Jiang, Integrated antimicrobial and nonfouling zwitterionic polymers. Angew. Chem. Int. Ed. 53, 1746-1754 (2014)

12. J. Mosnacek, A. Popelka, J. Osicka, J. Filip, M. Ilcikova, J. Kollar, A.B. Yousaf, T. Bertok, J. Tkac, P. Kasak, J. Colloid Interface Sci. 512, $511(2018)$

13. P. Sobolčiak, M. Špírek, J. Katrlík, P. Gemeiner, I. Lacík, P. Kasák, Light-switchable polymer from cationic to zwitterionic form: synthesis, characterization, and interactions with dna and bacterial cells. Macromol. Rapid Commun. 34, 635-639 (2013)

14. G.S. Asane, S.A. Nirmal, L.B. Rasal, A.A. Naik, M.S. Mahadik, Y.M. Rao, Polymers for mucoadhesive drug delivery system: a current status. Drug Dev. Ind. Pharm. 34, 1246-1266 (2008)

15. N.A. Peppas, P. Buri, Surface, interfacial and molecular aspects of polymer bioadhesion on soft tissues. J. Control. Release 2, 257-275 (1985)

16. J. Woodley, Bioadhesion. Clin. Pharmacokinet. 40, 77-84 (2001)

17. J.D. Smart, The basics and underlying mechanisms of mucoadhesion. Adv. Drug Deliv. Rev. 57, 1556-1568 (2005)

18. T. Klemetsrud, H. Jonassen, M. Hiorth, A.L. Kjoniksen, G. Smistad, Studies on pectin-coated liposomes and their interaction with mucin. Colloids Surf. B-Biointerfaces 103, 158-165 (2013)

19. M.L. Manca, S. Mourtas, V. Dracopoulos, A.M. Fadda, S.G. Antimisiaris, PLGA, chitosan or chitosan-coated PLGA microparticles for alveolar delivery? Colloids Surf. B-Biointerfaces 62, 220 $231(2008)$
20. T.A. Sonia, C.P. Sharma, N-hydroxypropyltrimethylammonium polydimethylaminoethylmethacrylate sub-microparticles for oral delivery of insulin - An in vitro evaluation. Colloids Surf. BBiointerfaces 107, 205-212 (2013)

21. F. Kianfar, M. Antonijevic, B. Chowdhry, J.S. Boateng, Lyophilized wafers comprising carrageenan and pluronic acid for buccal drug delivery using model soluble and insoluble drugs. Colloids Surf. B-Biointerfaces 103, 99-106 (2013)

22. C. Giovino, I. Ayensu, J. Tetteh, J.S. Boateng, An integrated buccal delivery system combining chitosan films impregnated with peptide loaded PEG-b-PLA nanoparticles. Colloids Surf. B-Biointerfaces 112, 9-15 (2013)

23. D. Duchene, F. Touchard, N.A. Peppas, Pharmaceutical and medical aspects of bioadhesive systems for drug administration. Drug Dev. Ind. Pharm. 14, 283-318 (1988)

24. R. Gurny, J.M. Meyer, N.A. Peppas, Bioadhesive intraoral release systems: design, testing and analysis. Biomaterials 5, 336-340 (1984)

25. E. Doelker, Cellulose derivatives. Adv. Polym. Sci. 107, 199 (1993)

26. B. Mizrahi, J. Golenser, J.S. Wolnerman, A.J. Domb, Adhesive tablet effective for treating canker sores in humans. J. Pharm. Sci. 93, 2927-2935 (2004)

27. Q. Zhang, D. Lin, S. Yao, Review on biomedical and bioengineering applications of cellulose sulfate. Carbohydr. Polym. 132, 311$322(2015)$

28. Z.M. Wang, K.J. Xiao, L. Li, J.Y. Wu, Molecular weight-dependent anticoagulation activity of sulfated cellulose derivatives. Cellulose 17, 953-961 (2010)

29. W.M. El-Sadr, K.H. Mayer, L. Maslankowski, C. Hoesley, J. Justman, F. Gai, et al., Safety and acceptability of cellulose sulfate as a vaginal microbicide in HIV-infected women. AIDS 20, 1109$1116(2006)$

30. N. Aggarwal, N. Altgärde, S. Svedhem, K. Zhang, S. Fischer, T. Groth, Study on multilayer structures prepared from heparin and semi-synthetic cellulose sulfates as polyanions and their influence on cellular response. Colloids Surf. B: Biointerfaces 116, 93-103 (2014)

31. N. Aggarwal, N. Altgärde, S. Svedhem, K. Zhang, S. Fischer, T. Groth, Effect of molecular composition of heparin and cellulose sulfate on multilayer formation and cell response. Langmuir 29, 13853-13864 (2013)

32. A. Vikartovská, M. Bucko, D. Mislovicová, V. Pätoprsty, I. Lacík, P. Gemeiner, Improvement of the stability of glucose oxidase via encapsulation in sodium alginate-cellulose sulfatepoly(methylene-co-guanidine) capsules. Enzym. Microb. Technol. 41, 748-755 (2007)

33. I. Lacík, M. Brissová, A.V. Anilkumar, A.C. Powers, T. Wang, J. Biomed. Mater. Res. 39, 52 (1997)

34. C.M. Lehr, F.C.G. Poelma, H.E. Junginger, J.J. Tukker, An estimate of turnover time of intestinal mucus gel layer in the rat in situ loop. Int. J. Pharm. 70, 235-240 (1991)

35. C. Valenta, The use of mucoadhesive polymers in vaginal delivery. Adv. Drug Deliv. Rev. 57, 1692-1712 (2005)

36. C. Valenta, C.E. Kast, I. Harich, A. Bernkop-Schnurch, Development and in vitro evaluation of a mucoadhesive vaginal delivery system for progesterone. J. Control. Release 77, 323-332 (2001)

37. H. Hägerström, K. Edsman, Interpretation of mucoadhesive properties of polymer gel preparations using a tensile strength method. J. Pharm. Pharmacol. 53, 1589-1599 (2001)

38. E.E. Hasan, J.M. Gallo, Pharm. Res. 7, 491-495 (1990)

39. F. Madsen, K. Eberth, J.D. Smart, A rheological examination of the mucoadhesive/mucus interaction: the effect of mucoadhesive type and concentration. J. Control. Release 50, 167-178 (1998)

40. H. Hägerström, M. Paullsson, K. Edsman, Evaluation of mucoadhesion for two polyelectrolyte gels in simulated 
physiological conditions using a rheological method. Eur. J. Pharm. Sci. 9, 301-309 (2000)

41. R.G. Riley, J.D. Smart, J. Tsibouklis, P.W. Dettmar, F. Hampson, J.A. Davis, G. Kelly, R.W. Wilber, An investigation of mucus/ polymer rheological synergism using synthesised and characterised poly(acrylic acid)s. Int. J. Pharm. 217, 87-100 (2001)

42. D. Pliszaczak, C. Bordes, S. Bourgeois, P. Marote, H. Zahouani, S. Tupin, C. Pailler Mattei, P. Lanteri, Colloids Surf. B-Biointerfaces 92, 168 (2012)

43. M.D. Hornof, C.E. Kast, A. Bernkop-Schnurch, In vitro evaluation of the viscoelastic properties of chitosan-thioglycolic acid conjugates. Eur. J. Pharm. Biopharm. 55, 185-190 (2003)

44. C. Caramella, M.C. Bonferoni, S. Rossi, F. Ferrari, Eur. J. Pharm. Sci. 40, 213-217 (1994)

45. D. Ivarsson, M. Wahlgren, Comparison of in vitro methods of measuring mucoadhesion: ellipsometry, tensile strength and rheological measurements. Colloids Surf. B-Biointerfaces 92, 353-359 (2012)

46. I. Ayensu, J.C. Mitchell, J.S. Boateng, Development and physicomechanical characterisation of lyophilised chitosan wafers as potential protein drug delivery systems via the buccal mucosa. Colloids Surf. B-Biointerfaces 91, 258-265 (2012)

47. S. Rossi, M.C. Bonferoni, G. Lippoli, M. Bertoni, F. Ferrari, C. Caramella, U. Conte, Influence of mucin type on polymer-mucin rheological interactions. Biomaterials 16, 1073-1079 (1995)

48. F. Madsen, K. Eberth, J.D. Smart, A rheological evaluation of various mucus gels for use in-vitro mucoadhesion testing. Pharm. Sci. 2, 563-566 (1996)

49. J. Kocevar-Nared, J. Kristl, J. Smid-Korbar, Comparative rheological investigation of crude gastric mucin and natural gastric mucus. Biomaterials 18, 677-681 (1997)
50. S.A. Mortazavi, J.D. Smart, Factors influencing gel-strengthening at the mucoadhesive-mucus interface. J. Pharm. Pharmacol. 46, 8690 (1994)

51. P.J. Serafinowski, P.B. Garland, Novel photoacid generators for photodirected oligonucleotide synthesis. J. Am. Chem. Soc. 125, 962-965 (2003)

52. S.A. Mortazavi, B.G. Carpenter, J.D. Smart, A comparative study on the role played by mucus glycoproteins in the rheological behaviour of the mucoadhesive/mucosal interface. Int. J. Pharm. 94, 195-201 (1993)

53. S.A. Mortazavi, B.G. Carpenter, J.D. Smart, An investigation of the rheological behaviour of the mucoadhesive/mucosal interface. Int. J. Pharm. 83, 221-225 (1992)

54. D.S. Jones, A.D. Woolfson, A.F. Brown, Textural, viscoelastic and mucoadhesive properties of pharmaceutical gels composed of cellulose polymers. Int. J. Pharm. 151, 223-233 (1997)

55. L. Styer, Biochemistry, Freeman \& Co, CA, USA, p. 44 (1975)

56. S. Duggan, O. O'Donovan, E. Owens, E. Duggan, H. Hughes, W. Cummins, Comparison of the mucoadhesive properties of thiolated polyacrylic acid to thiolated polyallylamine. Int. J. Pharm. 498, 245-253 (2016)

57. G. Sandri, S. Rossi, M.C. Bonferoni, F. Ferrari, M. Mori, C. Caramella, The role of chitosan as a mucoadhesive agent in mucosal drug delivery. J. Drug Del. Sci. Technol. 22, 275-284 (2012)

58. P.G. Andrews, T.P. Laverty, D.S. Jones, Mucoadhesive polymeric platforms for controlled drug delivery. Eur. J. Pharm. Biopharm. 71, 505-518 (2009)

59. J.-M. Schumers, C.-A. Fustin, A. Can, R. Hoogenboom, U.S. Schubert, J.-F. Gohy, Areo-nitrobenzyl (meth)acrylate monomers polymerizable by controlled-radical polymerization? J. Polym. Sci. A Polym. Chem. 47, 6504-6513 (2009) 